\title{
Benglish Verbs: a Case of Code-Mixing in Bengali*
}

\author{
Shishir Bhattacharja \\ Institute of Modern Languages, University of Dhaka \\ Ramna, Dhaka 1000, Bangladesh \\ shishir.bhattacharja@gmail.com
}

\begin{abstract}
In this article, we show how grammar can account for Benglish verbs, a particular type of complex predicate, which are constituted of an English word and a Bengali verb (e.g. /EksiDenT $\mathrm{kOra} /$ 'to have an accident', /in kOra/ 'to get/come/put in' or /kOnfuz kOra/ 'to confuse'). We analyze these verbs in the light of a couple of models (e.g. Kageyama, 1991; Lieber, 1992; Matsumoto, 1996) which claim that complex predicates are necessarily formed in syntax. However, Benglish verbs like /in kOra/ or / $\mathrm{kOnfuz} \mathrm{kOra/} \mathrm{are} \mathrm{problematic} \mathrm{for} \mathrm{these} \mathrm{approaches} \mathrm{because} \mathrm{it} \mathrm{is}$ unclear how preposition in or flexional verb confuse can appear as the arguments of the verb / $\mathrm{kOra} /$ 'to do' in an underlying syntactic structure. We claim that all Benglish verbs can be satisfactorily handled in Morphology in the light of Whole Word Morphology (Ford et al., 1997 and Singh, 2006).
\end{abstract}

Keywords: Benglish, Code-mixing, Complex predicates, Loan-verb Accommodation, WWM.

\section{Introduction}

As in many other languages (Butt, 1995, 2010; Dasgupta, 1977, 2003; Hook, 1974; Masica, 1976; Mohanan, 1993, 1994; Moravcsik, 1975, 1978 and Wohlgemuth, 2009 among others) there exists a particular type of complex predicates in Bengali constituted of two items, one chosen from among various categories of words: noun, verbal forms, adjective, preposition, adverb, onomatopoeic word, etc., and the other, a duly inflected verb. The first item is usually called a pole and second one a vector. These predicates are generally put into two different groups on the basis of the syntactic category of their pole: i) Compound verbs (1) that categorically involve a verb (usually a non-inflectional verbal form such as participle, absolutive or past gerund), and ii) Conjunct verbs (2) that involve categories other than the verb. The main characteristic of compound and conjunct verbs is that they must denote one single action.

1. Rik eshe poreche (Rik-having come-has fallen) 'Rik has just come.'

2. Rik bajar kore (Rik-market-does) 'Rik does shopping.'

\footnotetext{
* I am grateful to Professor Peter Hook and two anonymous reviewers for their comments and suggestions. I thank Professor Taro Kageyama, my post-doctorate research supervisor in Japan for the thought-provoking discussions I had with him concerning complex predicates and word formation. I thank my senior colleague Dr. Dil Afroze Quader who read several versions of this paper, gave comments, and tightened up my prose. Usual disclaimers apply. For financial support, I gratefully acknowledge the beneficence of the Japan Society for the Promotion of Science (JSPS) and University of Dhaka, Bangladesh, while for logistic support, I am indebted to the National Institute for Japanese Language and Linguistics, Tokyo, Japan.
}

Copyright 2010 by Shishir Bhattacharja 
As in other South Asian languages there is a particular type of complex predicate in Bengali in which the pole is a word of English origin chosen from among various types of nouns (3-6), adverbs (7), adjectives (8), prepositions (9) and verbs (10-11) while the vector is chosen from among a closed set of Bengali verbs consisting mainly of $/ \mathrm{kOra} /$ 'to do', /hOwa/ 'to be/to happen/to become', /dewa/ 'to give', /newa/ 'to take' and a few others. In this article, we will call these particular instances of code-mixing as Benglish verbs. As we can see in (3-11), some Benglish verbs are conjunct verbs (3-9), whereas some others (10-11) are compound verbs, which, unlike Bengali compound verbs, take a flexional verb as their pole (10-11).

3a. /EksiDenT kOra/ (accident-do) or 3b. EksiDenT hOwa (accident-be) 'to have an accident'

4. /ribhEnj newa/ (revenge-take) 'to take revenge'

5. /grup kOra/ (group-do) 'to put (things/persons) in a group'

6. /Ofish (or Ofis) kOra/ (office-do) 'to work in an office'

7. /slow kOra/ (slow-do) 'to make slow'

8. /kOmpaTibOl hOwa/ (compatible-be) 'to be compatible'

9. /in kOra/ (in-do) 'to get/come/put in'

10. /kOnfuz kOra/ (confuse-do) 'to confuse'

11. /jasTify kOra/ (justify-do) 'to justify'

If one takes the idea of the headedness of words as valid, one can note that like other complex predicates, Benglish verbs also manifest the head-final syntactic pattern of Bengali, not the head initial pattern of English. Hence, the pole precedes the vector in (4), whereas in the English match of the verb: /take revenge/, the pole /revenge/ follows the vector/take/. As with any other simple or complex predicates in Bengali each Benglish verb has its own subcategorical features. For example, /EksiDenT kOra/ (3a) and /EksiDenT hOwa/ (3b) - the two Benglish matches of the English verb 'to have an accident' require their agent nouns to be case-marked differently, the former with nominative (marked with zero affix) (12), and the latter with genitive (13).

12. Kawsar EksiDenT koreche

(Kawsar-accident-has done)

'Kawsar had an accident.'

13. Kawsarer EksiDenT hoyeche

(Kawsar-Gen-accident-has been)

'Kawsar had an accident.'

We note that many Benglish verbs such as /ofish kOra/ (6) and /in kOra/ (9) have no verbatim English counterparts. In some cases, speakers can alternate between a conjunct verb (e.g. /obhijog kOra/ (14)) and its Benglish counterpart (e.g. /kOmplein kOra/ (15)). However, not all Benglish verbs (e.g. /ofish kOra/ (6) and /kOmpaTibOl hOwa/ (7)) can be replaced with a Bengali conjunct verb, and in many cases, the English pole cannot alternate with its Bengali counterpart. For example, */durghOTona kOra/ cannot replace (3a) although /accident/ is frequently used as a synonym of /durghOTona/. On the other hand, /Eksident howa/ (3b) and /durghOtona howa/ are synonyms. 
14. Rik Fahimer kache Gargir biruddhe obhijog koreche (Rik-Fahim-Gen-near-Gargi-Gen-against-complain-did) 'Rik has complained to Fahim against Gargi.'

15. Rik Fahimer kache Gargir biruddhe kOmplein koreche (Rik-Fahim-Gen-near-Gargi Gen-against-complain-did) 'Rik has complained to Fahim against Gargi.'

In this article we will try to show how grammar can account for Benglish verbs. The present work is based on a quasi exhaustive list of about a thousand Benglish verbs. We presume that most of these verbs are used by a particular group of more of less urbanized Bengali speakers who are at different stages of bilingualism (in the sense of Singh and Backus, 2000:83). ${ }^{1}$ This article is organized as follows. In section 2 we demonstrate how Benglish verbs can be handled in the light of different models of morphology of our time which claim that complex predicates must be handled in syntax, and then we point out some examples that would be problematic for theses approaches. In section 3, after a brief description of $\mathrm{W}$ (hole) $\mathrm{W}$ (ord) $\mathrm{M}$ (orphology), we try to demonstrate how this model can account for all types of Benglish verbs in morphology. In section 4 we discuss some problems of handling Benglish verbs in the light of WWM, and finally, we draw conclusions.

\section{Benglish Verbs in the light of Different Models of Morphology}

In this section we will analyze Benglish verbs in the light of a couple of models that have been used to account for complex predicates in other languages. In Lieber (1983, 1992 and 2004), Benglish verbs will be treated as compounds. In her models, compound formation is constrained by the A(rgument) L(inking) P(rinciple) according to which a verb or a preposition must be able to link its internal arguments which Lieber (1983:257) defines, following Williams (1980), as follows: "all obligatory (i.e. lexically specified) arguments with the exception of the subject are internal." For instance, in (16), the dyadic verb /newa/ links its two internal arguments: experiencer /Gargi/ and theme /ribhEnj/, and in (17), the monadic verb/kOra/ links its only internal argument /kOmplein/. However, (17) is less acceptable than (15) because in English/to complain/ is a triadic verb which needs to link its own internal arguments such as experiencer (/Gargi/), goal (/Fahim/ $)^{2}$, etc.

16. Rik Gargir upOr ribhEnj niyeche

(Rik-Gargi-Gen-on-revenge-has taken)

'Rik has taken revenge on Gargi.'

17. ?Rik kOmplein koreche

(Rik-complain-has done)

'Rik has complained.'

The predicates like / $\mathrm{kOra} /$ 'to do' and /newa/ 'to take' can link their respective arguments in (36). However, it is unclear how ALP can be satisfied in (9-11) because categories like flexional

\footnotetext{
${ }^{1}$ Singh and Backus (2000:83) put bilinguals into two different groups: i) Perfect or true bilinguals and ii) less than perfect bilinguals. The latter are further divided into four subgroups: a. Very competent, b. Competent, c. Weak and d. Apparent.

${ }^{2}$ How weak and apparent bilinguals become aware of the subcategorical features of English verbs is a question left unanswered in this paper. We ignore whether they have a common lexicon for English and Bengali or full-fledged English verbs are part of their Bengali lexicon.
} 
verb (e.g. /kOnfuz/ and /jasTify/) and preposition (e.g. /in/) cannot usually function as arguments of some predicate (e.g. $/ \mathrm{kOra} /$ ). If the nominal forms of these verbs appeared as poles, for instance, */kOnfushOn/ (<confusion) kOra/ or */jasTifikeshOn (<justification) kOra/, then it would be easier to handle (10-11) under Lieber's models. This said, one may argue that English words like /in/, /kOnfuz/, /jasTify/, etc. are not listed as preposition or verbs in Bengali but as nouns. ${ }^{3}$ For instance, the English preposition /in/ (18) and the English verb /(to) complain/ (19) seem to be listed as nouns in Bengali because the $\mathrm{Cl}$ (assifier) /Ta/ can be concatenated to them. We note that a noun must be able to take a $\mathrm{Cl}$ in Bengali notwithstanding that all words that can take $\mathrm{Cl}$ must be a noun. ${ }^{4}$ However, as $/ \mathrm{kOnfuz} /$ and $/$ parTisipeiT/ shun $\mathrm{Cl}$ in (20-21), these words are probably not listed as nouns.

18. plein ranwEte inTa kokhOn korlo?

(aeroplane-runway-loc-in-Cl when did)

'When did the plane entered the runway?'

19. Fahim Riker kOmpleinTa kOnsiDar korbe

(Fahim-Rik-Gen-complaint-Cl-consider-will do)

'Fahim will consider Rik's complaint.'

20. *Rikke kOnfuzTa korbenna

(Rik-Acc-confuse-Cl-do-not)

'Don't confuse Rik.'

21. *Rik ei utshObe parTisipeiTTa korbena

(Rik-this-festival-Loc-participate-Cl-will not)

'Rik will not participate in this festival.'

On the basis of an analysis of Japanese conjunct verbs (such as /keikoku suru/ (warning-do) 'to warn') (22), Kageyama (1991:196), following Baker (1988), claims that such verbs are formed through a process called A(bstract) I(ncorporation) which he defines as "an instance of Incorporation that does not manifest any physical sign of movement but only gives abstract co-indexes to an incorporating host and an incorporated element". Hence, in an underlying syntactic structure (22), the V(erbal) N(oun) head (e.g. /keikoku/ 'warning') appears as the argument of the verb head /suru/ and the latter incorporates the former. For Kageyama (1991:196) AI is "basically a word-formation process, though it takes place in the syntax."

22. [John-wa $\mathrm{w}_{\mathrm{i}}$ [murabito-ni ookami-ga kuru-to-no $\left(\mathrm{pro}_{\mathrm{i}}\right)$ keikoku o] suru]

(John-Topi-villager-to-wolf-Nom-come-Comp Gen-( pro $_{\mathrm{i}}$ )-warn-Acc-does)

'John warns the villagers that the wolf is coming.

For Matsumoto (1996) Japanese conjunct verbs are bi-clausal constructions in which one predicate functions as the complement of the other while the subject of one predicate controls or binds (+ c-command, + co-indexation) the covert subject (pro) of the other predicate. Hence, in (22) the flexional verb /suru /and the $\mathrm{VN} / \mathrm{keikoku} /$ are two independent predicates, where the subject (John) of /suru/ controls the covert subject (pro) of /keikoku/. Similarly, in (23), the subject (Rik) of the vector / $\mathrm{kOra/} \mathrm{controls} \mathrm{the} \mathrm{covert} \mathrm{subject} \mathrm{(pro)} \mathrm{of} \mathrm{the} \mathrm{pole} / \mathrm{kOnfuz} /$.

\footnotetext{
${ }^{3}$ One may also argue that these words are underspecified in Bengali, or that they are 'neutral with regard to part-of-speech membership' (see Wolgemuth, 2009:102 cited later in this section), but how a non-noun can satisfy ALP remains a mystery.

${ }^{4}$ In examples like /tumi khabeTa ki/ (you-will eat-Ta-what) 'What (the hell) will you eat?' or /she geloTa kothay/ (she/he-went-Ta-where) 'Where (the hell) has she gone!' [Ta] is concatenated to a flexional verb.
} 
23. [Rik $\mathrm{R}_{\mathrm{i}}$ [tar bondhu-der $\left.\left(\mathrm{pro}_{\mathrm{i}}\right) \mathrm{kOnfuz}\right]$ kore]

(Rik-his-friend-Acc+Plu-( pro $_{\mathrm{i}}$ )-confuse-does)

'Rik confuses his friends.'

Both Kageyama (1991) and Matsumoto (1996) can satisfactorily account for the Benglish verbs in (3-6). However, (9-11) are problematic for these approaches because it is unclear how prepositions (9) or flexional verbs (10-11) can appear as the argument of the predicate $/ \mathrm{kOra} /$ in an underlying syntactic structure. In our view, examples like (9-11) are problematic for any model that attempts to account for word formation either in syntax or with syntactic rules.

For Wohlgemuth (2009:102) words like Benglish verbs would represent a particular way of L(oan) V(erb) A(ccommodation) which he calls, following Grimshaw and Mester (1988), Light Verb Strategy in which the borrowed verb (pole) remains mostly uninflected and more or less neutral with regard to part-of-speech membership. ${ }^{5}$ According to Wohlgemuth the vector verb has an auxiliary-like function and bears the inflection, while the semantic information is by and large associated with the pole. Wohlgemuth (2009) mentions two other important strategies of loan verb accommodation: i) Direct insertion (24) and ii) Indirect insertion (25-26). In direct insertion, the borrowed verb remains more or less unchanged, whereas in indirect insertion, they undergo morpho-syntactic adaptation.

\section{English Download $>$ German downloaden \\ 25. English Download > Indonesian downloadin \\ 26. English realize $>$ Hungarian realiz-ál}

Whether Wohlgemuth's (2009) LVA strategies are really different from each other is debatable. In the following section we will demonstrate that the whole phenomenon of LVA can be handled with one single strategy in the light of W(hole) W(ord) M(orphology).

\section{Benglish Verbs in the light of Whole Word Morphology}

In what follows, after a brief description of WWM (elaborated in Ford et al., 1997; Singh, 2006), we will try to demonstrate how Benglish verbs can be handled in this model. According to Singh (2006:578) "All that needs to be said about word structure in any language (of any type whatsoever) can and must be said by instantiations of the schema in (S1). These instantiations are referred to as Word Formation Strategies (WFSs) because, as generalizations drawn from known particular facts, they can be activated in the production and understanding of new words. WFSs must be formulated as generally as possible, but - and this is crucial - only as generally as the facts of the matter permit.

$\mathrm{S} 1 . / \mathrm{X} / \mathrm{a} \leftrightarrow / \mathrm{X}^{\prime} / \mathrm{b}$ where

1. $/ \mathrm{X} / \mathrm{a}$ and $/ \mathrm{X}^{\prime} / \mathrm{b}$ are words and $\mathrm{X}$ and $\mathrm{X}^{\prime}$ are abbreviations of the forms of classes of words belonging to categories $\mathrm{a}$ and $\mathrm{b}$ (with which specific words belonging to the right category can be unified or on to which they can be mapped).

2. ' represents (all the) form-related differences between $/ \mathrm{X} /$ and $/ \mathrm{X}^{\prime} /$ that fall outside of automatic phonology.

\footnotetext{
${ }^{5}$ According to Wohlgemuth (2009) light verb strategy is the second most frequently used strategy in world's languages and it can be found in all regions of the words and in most language families.
} 
3. $\mathrm{a}$ and $\mathrm{b}$ are categories that may be represented as feature bundles.

4. The $\leftrightarrow$ represents a bidirectional implication (if $/ X /$ then $/ X^{\prime} /$, and if $/ X^{\prime} /$, then $/ X /$ ).

5. The interpretation of $/ \mathrm{X} / \mathrm{a}$ is a semantic function of $/ \mathrm{X}^{1} / \mathrm{b}$ and vice versa.

6. ' can be null iff $\alpha \neq \beta$."

According to Singh (2006:578) "S1 offers a unified account of what have sometimes been seen as different types of morphologies and encapsulates the rejection of multipartite analysis of words into 'roots', 'affixes', 'stems', and so on, entries that are hard to define and harder to tell apart." For WWM, words have no internal (non-phonological) hierarchical structure. According to this model (Singh, 2006:578), "Morphological complexity is a matter of analyzability (\# segmentability) of a word into a variable and a constant component with respect to a WFS." WWM sees morphology, as Singh (2006:578) expresses it, "not as a combinatorics of morphs or morphemes but as a system of generalized and abstract bidirectional correspondence among patterns instantiated by sets of whole words that exploit the same contrast."

For example, on the basis of morphologically related sets of words like (27) and (28), one can obviously set up a WFS like (29). We note that (29) is licensed by a set of semantically related word-pairs that manifest the same i) formal contrast: $\mathrm{X} / \mathrm{Xli}$ on the one hand, and ii) categorical affiliation: Noun/Adjective on the other. According to (29) bad, kind, famous etc. provide the differing values for the variable $X$ in (27-28) while the phonemic representation /li/, which remains constant throughout (28), provides the particular value of the prime (') in the schema (S1).

\begin{tabular}{|c|c|c|}
\hline 27 & & 28 \\
\hline kind & $\leftrightarrow$ & kindly \\
\hline famous & $\leftrightarrow$ & famously \\
\hline bad & $\leftrightarrow$ & badly \\
\hline etc. & & etc. \\
\hline
\end{tabular}

29. $/ \mathrm{X} /{ }_{\mathrm{N}} \leftrightarrow / \mathrm{Xli} /$ Adj

We will now move onto demonstrate how WWM can account for Benglish verbs. We assume that the lexicon of a bilingual Bengali speaker contains sets of word-pairs constituted of English verbs like confuse or complain and Benglish verbs like /kOnfuzkOra/ or /kOmpleinkOra/ (30ab). Such pairs license WFSs like (30) which can be used to form, analyze and retrieve other Benglish verbs like /EnalaizkOra/ 'to analyze' or /jasTifykOra/ 'to justify'. Benglish verbs such as (9) can be obtained through (31). Note that there is no gap between the subcomponents of the right-hand outputs of (30-33) because we consider them single words.

30. $/ \mathrm{X} / \mathrm{v}$, Imp, 2nd $\leftrightarrow / \mathrm{XkOra} / \mathrm{v}$, Inf

a. /confuse/ $\leftrightarrow /$ kOnfuzkOra/ 'to confuse'

b. /complain/ $\leftrightarrow /$ kOmpleinkOra/ 'to complain'

c. /insult/ $\leftrightarrow$ /insalTkOra/ 'to insult'

d. /group/ $\leftrightarrow$ /grupkOra/ 'to put (things/persons) in a group'

31. $/ \mathrm{X} / \mathrm{P} \leftrightarrow / \mathrm{XkOra} / \mathrm{V}, \operatorname{Inf}$

/in/ $\leftrightarrow /$ inkOra/ 'to put (something) in'

/off/ $\leftrightarrow /$ OfkOra/ 'to put (something) off'

We note that pairs manifesting the same formal difference but different categorical affiliation (e.g. (30-31)) must license different WFSs. WFSs also differ from each other on the question of semantic relatedness. For example, although the formal difference and categorical affiliations 
are the same in (32-33) they are different WFSs because they are licensed with pairs based on different semantic relatedness.

32. $/ \mathrm{X} / \mathrm{N} \leftrightarrow / \mathrm{XkOra} / \mathrm{v}$, Inf

a. /EksiDenT/ 'accident' $\leftrightarrow /$ EksiDenTkOra/ 'to have an accident'

b. /insalT/ 'insult' $\leftrightarrow$ /insalTkOra/ 'to insult'

c. /obhijog/ 'complaint' $\leftrightarrow$ /obhijogkOra/ 'to complain'

d. /kOmplein/ 'complaint' $\leftrightarrow$ /kOmpleinkOra/ 'to complain'

e. /group/ $\leftrightarrow /$ grupkOra/ 'to put (things/persons) in a group'

33. $/ \mathrm{X} / \mathrm{N} \leftrightarrow / \mathrm{XkOra} / \mathrm{v}$, Inf

/Ofish/ 'office' $\leftrightarrow$ /OfishkOra/ 'to work in some office'

/kOlej/ 'college' $\leftrightarrow / \mathrm{kOlejkOra/} \mathrm{'to} \mathrm{attend} \mathrm{some} \mathrm{college'}$

We note in (32) that some Benglish verbs and their Bengali conjunct verb counterpart can be formed with the same WFS. Hence, Benglish /kOmplein kOra/ and Bengali /obhijog kOra/ can be obtained by mapping the nouns /kOmplein/ ( $<$ English Complaint) and /obhijog/ respectively onto (32). However, unlike English complain its Bengali match /obhijog/ cannot be mapped onto (30) because the latter is not a verb. We also note that some of the left-hand inputs of (30) (e.g. insult, group) have double categorical affiliation- they can be used either as a noun or as a verb in Bengali and hence, they can be mapped onto (31) as well. ${ }^{6}$

We mentioned in section 2 that according to Wohlgemuth (2009) there are four different strategies of LVA. If we follow the WWM approach, there will be but one strategy: WFS. We claim that most of the cases of LVA mentioned in the voluminous work by Wohlgemuth (2009) (about hundred languages belonging to about seventy language families) can be handled with relevant WFSs instantiating the schema in (S1). These 'code-mixed' words can also be analyzed into the variable instantiated by loan words and the constant by sequences like kora (kOmpleinkOra in Bengali), en (downloaden in German) in (downloadin in Indonesian) or ál (realizal in Hungarian) if we map them onto those WFSs.

Finally, we claim that there is no need to treat Benglish verbs in syntax, or to list each one of them in the lexicon of individual speakers. Vector verbs and their regular verb counterparts do not need to be listed separately either. However, some (but, not necessarily the same) Benglish verbs must be part of different individual lexicons, which together with relevant English words form adequate pairs that license different WFSs which can activated, in case of need, to form, retrieve or understand other Benglish verbs.

\section{Problems with the WWM Approach}

As with many compound and conjunct verbs in Bengali, one may argue that Benglish verbs are not words but phrasal constructions because some of them (e.g. 34-35) lack formal cohesion in the sense that their pole and the vector can be interrupted with some other words. This is why one of our anonymous reviewers states that Benglish verbs are 'single lexemes which consist of two words', and therefore, they are a 'very severe challenge for WWM'.

34. EksiDenT Kawsar kOkhon koreche?

(accident-Kawsar-when-did)

'When did Kawsar have an accident?'

\footnotetext{
${ }^{6}$ English nouns like 'insult or 'import differ from the verbs in'sult and im'port as regards the placement of stress. However, /insalT/ is pronounced unstressed in Bengali irrespective of whether it is a noun or a verb.
} 
35. kEarful Rikke oboshshoi thakte hObe (careful-Rik-Acc-certainly-remain-will be) 'Rik has to be careful.'

Linguists generally tend to agree that i) a large number of compounds derive from phrases and ii) many affixes derive from words that used to appear in compounds (Dressler 2006). We may divide this whole process of grammaticalization into four consecutive stages: i) Loose compounds (Dasgupta, 2003; Dressler, 2006) > ii) Tight or normal compounds > iii) Affixoidal words > iv) Affixal words. In the first stage, the two components of the compound in question may be interrupted by some other words, which is no more possible at the second stage. In the third stage, one of the components of the compounds undergoes phonological modification (such as Africa $>$ Afro, India >Indo, etc.) and becomes affixoid (Booij, 2004; Bauer, 2005) before finally becoming an affix (such as like $>l y$ ).

In some dialects of Bengali (e.g. Chittagonian (36) and Kishoreganj dialect (37)) there are examples of compound verbs that have been fused into simple verbs. We claim, on the basis of examples like (36-37) that verbs that appear as vector in Benglish verbs (and also in other compound and conjunct verbs) have already stepped into the process of grammaticalization, and are heading towards becoming something that are generally described as affixes (like dom in kingdom and hood in boyhood which were regular words at some point of diachrony), which however does not mean that all vector verbs must cease to be used as regular verbs in the long run.

\section{6. khai phalai (having eaten-I have thrown off) $>$ khaialai "I have finished eating'}

\section{7. khaia phalaichi (having eaten-I have thrown off) > khaialchi "I have finished eating'}

We are aware of the fact that (36-37) are neither Benglish verbs, nor are they words of the standard dialect of Bengali with which we are concerned here. However, (38-39) point to fact that not all Benglish verbs can be interrupted, which means that some of them have already acquired some sort of formal integrity. It is possible that the Benglish verbs that lack cohesiveness are in their first stage of grammaticalisation and those which cannot be interrupted are in the second stage. Hence, although a large number of Benglish verbs lack formal integrity, we can claim, on the basis of (36-39), that all of them must acquire it in the course of time.

38. *Rik kOnfuz bondhuder kore

(Rik-confuse-friend-Acc/Plu-does)

'Rik confuses his friends'

39.? plain runwayte in khOkhon korbe?

(aeroplane-runway-Loc-in-when-will do)

'When will the aeroplane enter the runway?

As with other compound and conjunct verbs, Benglish verbs are semantically opaque in the sense that they denote one single action. For example, /EksiDenT kOra/ does not mean two simultaneous or consecutive actions such as 'to have an accident' and 'to do something'. Equally /kOnfuz kOra/ either means i) 'to mix up things (or persons) in the speaker's mind which are otherwise distinct', or ii) 'to make someone else to mix up things (or persons)', the agent performing in both cases nothing but one single 'action of confusing'. In our view, semantic opacity is a more reliable and (probably) more universal criterion for word-hood as compared to formal integrity because some languages have been reported (Sadock 1998) to have words that allow insertion of lexical and grammatical elements. As Benglish verbs categorically lack semantic compositionality, they are more likely to be words and can be handled in morphology with WFSs à la WWM. 
Save a few exceptions, linguists generally tend to agree that complex predicates are not phrases. ${ }^{7}$ If one takes this stand as valid, then these constructions can be but words. However, one can also propose some intermediate category between phrase and word such as $\mathrm{Word}^{+}$(Kageyama, 2001), or, following Mohanan (1994), put words into different subcategories such as 'morphological word' (e.g. complex predicates) and 'phonological word'. But, as long as the word-hood of the Benglish verbs is not challenged we do not see why they should be a challenge for WWM.

\section{Conclusions}

In this article we have studied Benglish verbs, a particular type of complex predicates in Bengali which are constituted of an English word and a Bengali verb, in the light of different models of morphology of our time. We have tried to demonstrate why a large number of these verbs are problematic for models that usually accounts for complex predicates in syntax and how all of them can be satisfactorily handled in morphology in the light of WWM.

Finally, it needs to be said that although we have used traditional terms like compound, compound verb, conjunct verb, complex predicate, pole, vector, light verbs, head, etc. throughout this article to ease discussion, there will, in fact, be no need of this if we adopt the WWM framework. We claim that all so-called simple and complex predicates including Benglish verbs are verbs and like any other verb (or any other word) they can be formed, analyzed or retrieved with the relevant WFSs.

Abbreviations: Acc: Accusative; Adj: Adjective; AI: Abstract incorporation; ALP: Argument linking principle; Cl: Classifier; Imp: Imperative; Gen: Genitive; Inf: Infinitive; LVA: Loan verb accommodation; Loc: Locative; N: Noun; P: Preposition; Plu: Plural; pro: covert subject pronoun; Top: Topicalizer; Nom: Nominative; V: Verb; VN: Verbal noun; WFS: Word formation strategy; WWM: Whole word morphology; $\mathbf{2}^{\text {nd }}$ : Second person.

\section{References}

Baker, M. 1988. Incorporation: a Theory of Grammatical Function Changing. Chicago: The University of Chicago press.

Bauer, L. 2005. The Borderline between Derivation and Compounding. In W. U. Dressler, D. Kastovsky, O. E. Pfeiffer, and F. Rainer, eds., Morphology and its Demarcations, pp. 97-107. Amsterdam: Benjamins.

Booij, G. 2004. Compounding and Derivation, Evidence from Construction Morphology. In Dressler et al., eds., Morphology and its Demarcations, pp. 109-131. Amsterdam: Benjamins.

Butt, M. 2010. The Light Verb Jungle: Still Hacking Away. In M. Amberber, B. Baker and M. Harvey, eds., Complex Predicates: Cross Linguistic Perspectives on Event Structure, pp. 4978. Cambridge: Cambridge University Press.

Butt, M. 1995. The Structure of Complex Predicate in Urdu. Stanford: CSLI.

Dasgupta, P.1977. The Internal Grammar of Compound Verbs in Bangla. Indian linguistics, 38, 68-77.

Dasgupta, P. 2003. Bangla. In G. Cardona and D. Jain, eds., The Indo-Aryan Languages, pp. 351-390. London: Routeledge.

Dressler, W. U. 2006. Compound Types. In G. Libben and G. Jarema, eds., The Representation and Processing of Compound Words, pp. 23-44. New York: Oxford University Press.

\footnotetext{
${ }^{7}$ For example, Grimshaw and Mester (1988:213) claim that the formation of Japanese conjunct verbs can be "assimilated to a more general theory of phrasal constructions, which governs the behavior of idioms (like kick the bucket) and other lexical expressions that do not constitute single words."
} 
Ford, A, R. Singh and G. Martohardjono. 1997. Pace Panini, towards a Word-based Theory of Morphology. New York: Peter Lang.

Grimshaw, J. and A. Mester (1988) Light verbs and A-marking. Linguistic Inquiry, 19(2):205-232.

Hook, P. E. 1974. The Compound Verb in Hindi. Michigan: Center for South and Southeast Asian Studies, The University of Michigan.

Kageyama, T. 1991. Light Verb Constructions and the Syntax-Morphology Interface. In H. Nakazima, ed., Current English Linguistics in Japan, pp. 169-203. Berlin: Mouton de Gruyter.

Kageyama, T. 2001. Word Plus: The Interaction of Words and Phrase. In J. V. D. Weijer and T. Nishihara, eds., Issues in Japanese Phonology and Morphology, pp. 245-270. Berlin: Mouton de Gruyter.

Lieber, R. 1983. Argument Linking and Compounds in English. Linguistic Inquiry,14 (2):251-285.

Lieber, R. 1992. Deconstructing Morphology: Word Formation in Syntactic Theory. Chicago: The University of Chicago Press.

Lieber, R. 2004. Morphology and Lexical Semantics. Cambidge: Cambridge University Press.

Masica, C. P. 2005 [1976]) Defining a Linguistic Area: South Asia. Chicago: The University of Chicago Press.

Matsumoto, Y. 1996. Complex Predicates in Japanese, a Syntactic and Semantic Study of the Notion 'Word'. California: CSLI Publications and Tokyo: Kurosio Publishers.

Mohanan, T. 1993. Verb Agreement in Complex Predicates in Hindi. In M. K. Verma, ed. Complex Predicates in South Asian languages, pp.163-175. New Delhi: Manohar publishers.

Mohanan, T. 1994. Argument Structure in Hindi. Stanford: CSLI.

Moravcsik, E. 1975. Verb Borrowing. Wiener Linguistiche Gazette, 8:3-30.

Moravcsik, E. 1978. Language Contact. In J. Greenberg, ed., Universals of Human Languages, Vol. 1. Method and Theory, pp. 93-122. Stanford: Stanford University Press,

Sadock, J. M. 1998. On the Autonomy of Compounding Morphology. In S. G. Lapointe, D. K. Brentari and P. M. Farrel, eds., Morphology and its Relation to Phonology and Syntax, pp. 161-187. California: CSLI Publications.

Singh, R. 2006. Whole Word Morphology. Elsevier Encyclopedia of Linguistics, 2nd edition, pp. 578-579.

Singh R. and A. Backus. 2000. Code-Mixing and Bilingual Proficiency. The Yearbook of South Asian Languages and Linguistics 2000, 61-92.

William, E. 1980. Argument Structure and Morphology, MS. University of Massachusetts at Amherest.

Wohlgemuth, J. 2009. A Typology of Verbal Borrowings. Berlin: Mouton de Gruyter. 